\section{Fette unproblematisch - Megastudie relativiert aktuelle Ernährungsempfehlungen}

\begin{abstract}
Eine fettarme Ernährungsweise ist nach den Ergebnissen einer großen weltweiten Ernährungsstudie nicht mehr zu empfehlen. Wider Erwarten wurde das Sterberisiko durch eine hohe Zufuhr an gesättigten Fettsäuren sogar gesenkt. Drei bis vier Portionen Obst und Gemüse sind offenbar ausreichend.
\end{abstract}

$D^{i}$ ie aktuellen Ernährungsempfehlungen müssen nach den neuesten Erkenntnissen aus der PURE-Studie wohl über Bord geworfen werden. Den Gesamt-Fettanteil auf weniger als $30 \%$ der Energiezufuhr zu beschränken, wirkt demnach nicht lebensverlängernd. Auch die seit langem postulierte Empfehlung, wenig gesättigte Fettsäure zu konsumieren, kann offenbar ad acta gelegt werden; derzeit wird ein Anteil von weniger als $10 \%$ der Energiezufuhr empfohlen.

\section{Über 100.000 Teilnehmer}

Für die PURE-Studie wurden 135.335 Menschen aus 18 Ländern in sieben Regionen der Welt (Nord- und Südamerika, Europa, mittlerer Osten, Südasien, China, Südostasien und Afrika) zu ihren Ernährungsgewohnheiten befragt und über einen Zeitraum von 7,4 Jahren nachbeobachtet.

Hierbei stellte sich heraus, dass diejenigen, deren Ernährung einen hohen Fettanteil enthielt, deutlich länger lebten als jene mit einer niedrigen Fettzufuhr (höchstes vs. niedrigstes Quintil). Die Gesamtmortalität lag signifikant um $23 \%$ niedriger. Selbst ein erhöhter Konsum von gesättigten Fettsäuren ging mit einer $14 \%$ geringeren Sterblichkeit einher, bei einfach gesättigten Fettsäuren war diese um $19 \%$ und bei mehrfach gesättigten Fettsäuren um 20 \% geringer.

Eine hohe Zufuhr von Kohlenhydraten ging dagegen mit einem um $28 \%$ erhöhten Sterberisiko einher.

Aktuelle Empfehlungen hinterfragt Dr. Andrew Mente hält die aktuellen Ernährungsempfehlungen aufgrund dieser Ergebnisse für fragwürdig. „Wir sollten uns von der Fettrestriktion lösen", kommentiert der der am McMaster University in Hamilton tätige Epidemiologe, der an der Studie mitgewirkt hat. Menschen mit einem hohen Kohlenhydrat- anteil in der Ernährung ( $>60 \%$ ) sollten diesen auf ein gemäßigtes Maß reduzieren. Von einem hohen Fettanteil gehe dagegen keine Gefahr aus. Von fettreduzierten Lebensmitteln rät Mente ab.

\section{Obst/Gemüse bieten Schutz}

Ein weiterer Aspekt, der in der PUREStudie untersucht wurde, war der Zusammenhang zwischen dem Konsum von Obst, Gemüse und Hülsenfrüchten und der Gesamt- sowie der kardiovaskulären Mortalität. Bei drei bis vier Portionen pro Tag (ca. 375 g) war das Sterberisiko um $22 \%$ geringer als bei einer Zufuhr von weniger als einer Portion täglich. Eine noch höhere Zufuhr brachte keinen weiteren Zugewinn an Lebenszeit.

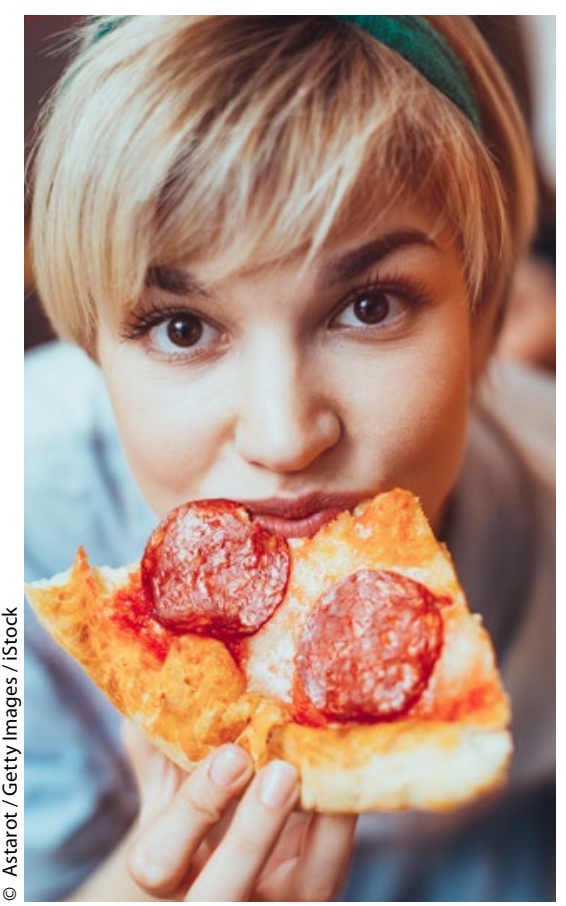

Die fettige Salami kann man in vollen Zügen genießen, zumindest das Sterberisiko erhöht diese Ernährungsweise nicht.
Bisher raten die Leitlinien mindestens fünf Portionen Obst, Gemüse oder Hülsenfrüchte ( $\geq 400 \mathrm{~g}$ ) zu sich zu nehmen. Eine solche Konsummenge konnte nur die Bevölkerung aus Nordamerika, Europa, Südamerika und dem mittleren Osten einhalten. Nach diesen Ergebnissen könnten aber schon drei bis vier Portionen ausreichen, meint Mente. Gerade in weniger entwickelten Ländern sei eine solche Menge wahrscheinlich realistischer zu erreichen.

Wie der Epidemiologe weiter ausführte, scheint nach diesen Daten rohes Gemüse gesundheitsförderlicher zu sein als gekochtes.

Erstaunlicherweise war der Einfluss einer ballaststoffreichen Ernährung auf die kardiovaskuläre Mortalität nur gering und statistisch nicht signifikant (Hazard Ratio: 0,89). Ein hoher Fettund Kohlenhydratanteil wirkte sich ebenfalls weder vor- noch nachteilig aus.

\section{Kardiovaskuläre Sterblichkeit} bleibt unbeeinflusst - warum?

Wie ist lässt sich dann die Reduktion der Gesamtmortalität erklären? Nach Ansicht der Studienleiter Dr. Mahshid Dehghan von der McMaster University könnte eine Ernährungsweise auch die Prävalenz anderer Erkrankungen wie Krebs- und Atemwegserkrankungen beeinflusst haben. Ein Einfluss von Störfaktoren schließt die Epidemiologin weitestgehend aus. „Wir haben auf alle bekannten Störfaktoren adjustiert und hoffen daher, dass es keine Verzerrung der Ergebnisse gegeben hat."

Trotz der Größe ist auch diese Studie aufgrund ihres beobachtenden Designs nicht ohne Limitationen. Die Angaben zur Ernährung wurden nur zu Studienbeginn erfasst. Es gibt keine Angaben zur Zubereitungsweise der Nahrungsmittel, also ob frittiert oder gebraten usw. Und auch der Anteil der als besonders schädlich geltenden Transfette wurde nicht erfasst.

Veronika Schlimpert

Quelle: Hot Line: Late-Breaking Registry Results

1 „Fruit, vegetable, and legume intake and cardiovascular disease and deaths: The Prospective Urban Rural Epidemiology (PURE) study of 135,000 people in 18 countries" und "Dietary fats are protective but carbohydrates are harmful: First results of the PURE nutrition study on 135,000 people from 18 countries in 5 continents", ESC-Kongress, 29.8.2017 in Barcelona 